Pacific Journal of Mathematics

NORM-ATTAINMENT OF LINEAR FUNCTIONALS ON
SUBSPACES AND CHARACTERIZATIONS OF TAUBERIAN

Richard Dean Neidinger and Haskell Paul Rosenthal 


\title{
NORM-ATTAINMENT OF LINEAR FUNCTIONALS ON SUBSPACES AND CHARACTERIZATIONS OF TAUBERIAN OPERATORS
}

\author{
RichaRd NeIdinger AND Haskell P. Rosenthal
}

It is proved that for every non-zero continuous linear functional $f$ on a non-reflexive Banach space $X$, there is a closed linear subspace $Y$ so that $\left.f\right|_{Y}$ does not attain its norm. In fact, $Y$ may be chosen with $\left\|\left.f\right|_{Y}\right\|$ arbitrarily close to $\|f\|$. It is also shown that every continuous linear functional on an infinite-dimensional normed linear space fails to attain its norm on some linear subspace. The class of non-zero Banach space operators which map closed bounded convex sets to closed sets is identified as the class of Tauberian operators. (A bounded linear operator $T: X \rightarrow Y$ is defined to be Tauberian provided $T^{* *} x^{* *} \in Y$ implies $x^{* *} \in X$.) Other closed image characterizations are obtained. In particular, using the very first result stated above, a non-zero operator is found to be Tauberian if and only if the image of the ball of any closed subspace is closed. The new characterizations show that the "hereditary versions" of semi-embeddings and $F_{\sigma}$-embeddings are precisely the one-to-one Tauberian operators.

Introduction. Let $X$ and $Y$ be Banach spaces and $T: X \rightarrow Y$ a given non-zero operator. (Throughout, "operator" means "bounded linear map".) Under what circumstances is it true that $T K$ is closed for every closed bounded convex subset $K$ of $X$ ? Evidently this is trivially true if $X$ is reflexive, so suppose this is not the case. Here are some of the equivalences obtained in our main result of $\$ 2$, Theorem 2.3. (For any Banach space $Z$, let $B_{Z}=\{z \in Z:\|z\| \leq 1\}$.)

Theorem. Assume $T: X \rightarrow Y$ is non-zero with $X$ non-reflexive. The following are equivalent:

(a) $T$ is Tauberian.

(b) $T K$ is closed for all closed bounded convex $K$.

(c) $T B_{Z}$ is closed for all closed linear subspaces $Z$.

(d) $T X$ is infinite-dimensional and $T Z$ is an $F_{\sigma}$ for all closed linear subspaces $Z$.

We recall the definition that $T: X \rightarrow Y$ is Tauberian provided whenever $G \in X^{* *}$ and $T^{* *} G \in Y$, then $G \in X$ (where we regard $X$ as canonically embedded in $X^{* *}$ ). Results of Kalton and Wilansky concerning Tauberian 
operators $[\mathbf{K W}]$ are reviewed at the beginning of $\S 2$. (Also, our results hold for either real or complex Banach spaces; for the sake of convenience, we treat the real case only.)

Recall that $T: X \rightarrow Y$ is a semi-embedding provided $T$ is one-to-one and $T B_{X}$ is closed. It is a result of Saint-Raymond (see [BR]) that $T$ is a semi-embedding under an equivalent norm on $X$ if (and only if) $T$ is one-to-one and $T X$ is an $F_{\sigma}$. The above result yields immediately that one-to-one operators are hereditarily semi-embeddings (under equivalent norms) if and only if they are Tauberian. That is, we have the

Corollary. Let $T: X \rightarrow Y$ be a one-to-one operator. The following are equivalent:

(a) $T$ is Tauberian.

(b) $\left.T\right|_{Z}$ is a semi-embedding for all closed linear subspaces $Z$ of $X$.

(c) $T Z$ is an $F_{\sigma}$ for all closed linear subspaces $Z$ of $X$.

Thus the class of Tauberian semi-embeddings is much smaller than that of semi-embeddings. It seems worth pointing out also that a semi-embedding $T$ is Tauberian if and only if $T^{* *}$ is one-to-one.

Actually, Tauberian semi-embeddings (i.e., one-to-one Tauberian operators) arise rather naturally in the study of Tauberian operators themselves. Indeed, let $T: X \rightarrow Y$ be a non-zero operator and $Z=\{x \in X$ : $T x=0\}$, the null space of $T$. Let $\Pi: X \rightarrow X / Z$ be the quotient map and $\tilde{T}: X / Z \rightarrow Y$ the natural map so that $T=\tilde{T} \Pi$. Then it is easily seen that $T$ is Tauberian if and only if $Z$ is reflexive and $\tilde{T}$ is a Tauberian semi-embedding. (We don't use this result in the sequel.) the role of Tauberian semi-embeddings in the factorization results in [DFJP] is discussed in $§ 2$.

Evidently the above Theorem yields that non-zero finite rank operators on a non-reflexive Banach space are never Tauberian. In particular, condition (c) of the Theorem yields that non-zero linear functionals on a non-reflexive space always have a non-norm-attaining restriction. The first section is essentially devoted to this result. Thus, we establish in Theorem 1.1 that if $X$ is nonreflexive and $f \in X^{*}$ is non-zero, there is a closed linear subspace $Z$ of $X$ so that $\left.f\right|_{Z}$ does not attain its norm and moreover $\left.f\right|_{Z}$ has norm arbitrarily close to that of $f$ itself. Of course this result is related to the discovery of James that there is a non-norm-attaining linear functional on every non-reflexive space. In fact, our argument for Theorem 1.1 uses the theorem of James [J1] that every weakly closed bounded set which is not weakly compact admits a functional whose sup on the set 
is not attained. Our original proof of Theorem 1.1 was significantly simplified by $\mathrm{N}$. Kalton who also observed that the non-norm-attaining restriction may always be chosen to be a restriction to a co-dimension one subspace. We thank Professor Kalton for his permission to include these improvements in this paper.

We also obtain that if $X$ is non-reflexive, then if $f \in X^{*}$ with $\|f\|=1$, there is a closed linear subspace $Z$ of $X$ with $\left\|\left.f\right|_{Z}\right\|=1$ and $\left\|\left.f\right|_{Z}\right\|$ not attained if (and only if) there is a $G \in X^{* *} \backslash X$ with $\|G\|=1=G(f)$. (Again, Kalton's argument yields that $Z$ may be chosen of co-dimension one.) Evidently Theorem 1.1 yields a characterization of non-reflexive Banach spaces. If we drop the condition that $Z$ be closed, we simply obtain a characterization of infinite-dimensional spaces. That is, we obtain in Theorem 1.2 that for any infinite-dimensional normed linear space and any non-zero linear functional on it, the restriction to some linear subspace has norm equal to the norm on the entire space and the norm is not attained.

A somewhat weaker result than Theorem 2.3 (the first Theorem presented in this introduction) is presented in [NR]. There we obtained the equivalence of conditions (a), (b), and (d) but lacked the full strength of condition (c). In order to obtain (c), the results in $\$ 1$ of this paper were formulated and their proofs discovered.

1. The non-norm-attainment of linear functionals on subspaces. This section contains the results about restricting linear functionals so that the norm of the restriction is not attained, as outlined in the introduction. Throughout this paper, the word "subspace" will refer to a closed linear subspace except (as noted) in the proof and discussion of Theorem 1.2. Also any functional is understood to be continuous and linear. We let $\langle f\rangle$ denote the linear span of $\{f\}$.

We now present the main result of this section. The proof employs mostly standard functional analysis. Part of this argument requires the fundamental theorem of James (in [J]1) which states: if $W$ is a bounded weakly closed subset of a Banach space and $W$ is not weakly compact, then there is a continuous linear functional that does not attain its supremum on $W$.

THEOREM 1.1. Let $B$ be a non-reflexive Banach space, $f \in B^{*}$ with $f \neq 0$, and $0<\lambda \leq\|f\|$. Suppose either

(a) $\lambda<\|f\|$

or

(b) $\lambda=\|f\|$ and there exists $a G \in B^{* *} \backslash B$ with $\|G\|=1$ and $G(f)$ $=\|f\|$. 
Then there exists a closed linear subspace $Y$ of co-dimension 1 in $B$ so that $\left\|\left.f\right|_{Y}\right\|=\lambda$ and $\left.f\right|_{Y}$ does not attain its norm.

REMARK. Evidently the final assertion of the Theorem means that $f\left(B_{Y}\right)=\{\alpha \in \mathbf{R}:|\alpha|<\lambda\}$.

Proof. We assume without loss of generality that $\|f\|=1$. Let $W=\{x \in B:\|x\| \leq 1$ and $f(x)=\lambda\}$, a closed bounded convex subset of $B$. We make the following claim: there exist $g \in B^{*} \backslash\langle f\rangle$ and $G \in B^{* *}$ such that $\|G\| \leq 1, G(f)=\lambda$, and $G(g)>g(w)$ for all $w \in W$.

Before we prove this claim, let us assume it and complete the proof. Let $h=g-(G(g) / \lambda) f$, then $G(h)=0$. Let $Y=N(h)$, the null space or pre-annihilator of $h$. Since $g \notin\langle f\rangle, h \neq 0$ and $Y$ is clearly a subspace of co-dimension 1 in $B$. Now $Y^{\perp \perp}=\langle h\rangle^{\perp}$ and hence $G \in Y^{\perp \perp}$. Identifying $Y^{* *}$ with $Y^{\perp \perp}$, it follows that $\left\|\left.f\right|_{Y}\right\| \geq G(f)=\lambda$. Suppose there exists a $y \in Y$ such that $\|y\| \leq 1$ and $f(y) \geq \lambda$. Let $w=(\lambda / f(y)) y$, then $w \in W$ $\cap Y$. Since $h(w)=0, g(w)=(G(g) / \lambda) f(w)=G(g)$, but this contradicts the claim. Thus $f(y)<\lambda$ for all $y \in B_{Y}$ meaning that $\left\|\left.f\right|_{Y}\right\|=\lambda$ and this norm is not attained.

We now prove the claim. First suppose $W$ is empty. By the Hahn-Banach Theorem, there is a $G \in B^{* *}$ such that $\|G\| \leq 1$ and $G(f)=\lambda$. Let $g$ be any element of $B^{*} \backslash\langle f\rangle$ and the claim holds. (Actually, $W=\varnothing$ implies $\lambda=1$ and $f$ does not attain its norm on $B$ itself.) Now assume $W$ is non-empty. Note that the condition $g \notin\langle f\rangle$ is superfluous. Indeed, if $g=\alpha f$ for some $\alpha$ and $w \in W$ then $G(g)=\alpha \lambda=$ $g(w)$, contradicting the condition $G(g)>g(w)$ for all $w \in W$. We now consider two cases depending on whether or not $W$ is weakly compact.

Case (a). Assume $W$ is weakly compact. We first establish that there is a $G \in B^{* *} \backslash B$ such that $\|G\| \leq 1$ and $G(f)=\lambda$. If $\lambda=1$, the hypothesis explicitly assumes that such a $G$ exists. If $\lambda<1$, choose $\delta>0$ sufficiently small depending on $\lambda$. Let $H \in B^{* *} \backslash B$ with $\|H\| \leq \delta$. Let $x_{0} \in B$ such that $\left\|x_{0}\right\|=1$ and $f\left(x_{0}\right) \geq 1-\delta$. Let $G=\lambda\left(x_{0}+H\right) /\left(f\left(x_{0}\right)+H(f)\right)$. Clearly $G \notin B$ and $G(f)=\lambda$. Also,

$$
\|G\| \leq \frac{\lambda\left(\left\|x_{0}\right\|+\|H\|\right)}{\left|f\left(x_{0}\right)\right|-|H(f)|} \leq \frac{\lambda(1+\delta)}{(1-\delta)-\delta} .
$$

Thus if $\delta$ is chosen so that $(1+\delta) /(1-2 \delta) \leq 1 / \lambda$, then $\|G\| \leq 1$.

Consider $W$ as a subset of $B^{* *}$. By the case (a) assumption, $W$ is a weak*-compact, convex subset of $B^{* *}$ and $G \notin W$ (since $W \subset B$ ). By a 
Hahn-Banach separation in the locally convex topological linear space $B^{* *}$ endowed with the weak* topology, there exists a weak*-continuous linear functional $g$ on $B^{* *}$ such that $g(G)>g(w)$ for all $w \in W$. Since $g$ is weak*-continuous, $g \in B^{*}$ and the claim is established in case (a).

Case (b). Assume $W$ is not weakly compact. By the fundamental theorem of James $\left[\mathbf{J 1}\right.$ ], we may choose a $g \in B^{*}$ so that $M=\sup g(W)$ is not attained on $W$. Now there exists a $G \in \tilde{W}$, the weak*-closure of $W$ in $B^{* *}$, with $G(g)=M$. Thus $G(g)>g(w)$ for all $w \in W$. Also $G \in \tilde{W}$ implies $\|G\| \leq 1$ and $G(f)=\lambda$. This completes the entire proof.

Theorem 1.1 also characterizes those linear functionals which have a non-norm-attaining restriction with norm equal to the norm on the entire space.

COROLlaRY. Let $f$ be a non-zero linear functional on $B$. There exists a closed linear subspace $Y$ of $B$ such that $\left.f\right|_{Y}$ does not attain its norm and $\left\|\left.f\right|_{Y}\right\|=\|f\|$ if and only if there is $a G \in B^{* *} \backslash B$ with $\|G\|=1$ and $G(f)=\|f\|$.

Proof. One direction is proved in Theorem 1.1. Suppose conversely that $Y$ is a subspace, $\left\|\left.f\right|_{Y}\right\|=\|f\|$ and $\left.f\right|_{Y}$ does not attain its norm. By the Hahn-Banach Theorem, there exists a $G \in Y^{* *}$ with $\|G\|=1$ and $G(f)=\|f\|$. Since $\left.f\right|_{Y}$ does not attain its norm, $G \notin Y$. Identifying $Y^{* *}$ with a subspace of $B^{* *}, G \in B^{* *} \backslash B$.

There are linear functionals where such a $G$ (equivalently, such a restriction) does not exist. Indeed, consider $B=l^{1}$ and let $f$ be the first coordinate functional, i.e., $f(x)=x(1)$, for all $x \in l^{1}$. If $Y$ is a subspace with $\left\|\left.f\right|_{Y}\right\|=1$, then there exists a sequence $\left(y_{i}\right)$ in $Y$ where $\left\|y_{i}\right\| \rightarrow 1$ and $f\left(y_{i}\right) \rightarrow 1$. This means that $\left|y_{i}(1)\right| \rightarrow 1$ and $\sum_{n=2}^{\infty}\left|y_{i}(n)\right| \rightarrow 0$ so that $y_{i} \rightarrow e_{1}$, the first unit vector. Hence $\left\|\left.f\right|_{Y}\right\|$ is attained at $e_{1} \in Y$. In general, a linear functional is strongly exposing on the unit ball (cf. [B], p. 269) if and only if $\left\|y_{i}\right\| \rightarrow 1$ and $f\left(y_{i}\right) \rightarrow\|f\|$ imply $\left(y_{i}\right)$ converges in norm. Therefore any strongly exposing linear functional attains its norm on any subspace $Y$ with $\left\|\left.f\right|_{Y}\right\|=\|f\|$.

Theorem 1.1 states that, for any $0<\lambda<\|f\|$, a subspace $Y$ always exists where $\left\|\left.f\right|_{Y}\right\|=\lambda$ and this norm is not attained (assume $B$ is non-reflexive). Examples such as the one above show that this is the best possible in the sense that a non-norm-attaining restriction with $\lambda=\|f\|$ may not exist. 
On the other hand, an example of an $f$ which does have a non-normattaining restriction with norm equal to $\|f\|$ is the limit functional on the space of convergent sequences. Let $B=c=C(\mathbf{N} \cup\{\infty\})$ and use the notation $x(\infty)=\lim _{i \rightarrow \infty} x(i)$ for all $x \in c$. Let $f$ be the norm-one functional $f(x)=x(\infty)$ for all $x \in c$. Then $c^{*}=l^{1}(\mathbf{N} \cup\{\infty\})$ and $c^{* *}=$ $l^{\infty}(\mathbf{N} \cup\{\infty\})$. Let $G \in c^{* *}$ be defined by $G(\infty)=1$ and $G(i)=0$ for all $i$. Clearly $G \notin c$ and $G(f)=1=\|G\|$. Thus by Theorem 1.1, there is a subspace $Y$ where $\left\|\left.f\right|_{Y}\right\|=1$ and this norm is not attained; this subspace is not explicitly obvious.

In Theorem 1.1, it is crucial that the constructed linear subspace be closed. In fact, the following theorem shows that if $X$ is a normed linear space and $f \in X^{*}$, then there exists a linear subspace $Y$ such that $\left\|\left.f\right|_{Y}\right\|$ is not achieved if and only if $X$ is infinite-dimensional. Moreover, one can choose $Y$ so that $\left\|\left.f\right|_{Y}\right\|=\|f\|$. James also considered a normed linear space version of his fundamental theorem that in any non-reflexive Banach space, there exists a linear functional not attaining its norm. One might ask if such a functional exists on any incomplete normed linear space. A counterexample is given in [J2], i.e., an incomplete normed linear space where every linear functional attains its norm. However in our setting, given any linear functional, it is always possible to find a restriction to a (not necessarily closed!) linear subspace on which the norm is not attained.

THEOREM 1.2. Let $X$ be an infinite-dimensional normed linear space and $f \in X^{*}$ with $\|f\|=1$. Then there exists a linear subspace $Y$ such that $\left\|\left.f\right|_{Y}\right\|=1$ and $\left.f\right|_{Y}$ does not attain its norm.

It is clear that Theorem 1.2 follows from inductive application of the following lemma. $X$ and $f$ remain as in Theorem 1.2.

LEMMA 1.3. If $E$ is a finite-dimensional subspace of $X$ such that $\left\|\left.f\right|_{E}\right\|<1$, then for any $\varepsilon>0$, there is a $y$ in $X$ such that $f(y)=1$, $\|y\|<1+\varepsilon$, and $\left\|\left.f\right|_{E+\langle y\rangle}\right\|<1$.

It will be shown that this lemma, in turn, follows from the following finite-dimensional lemma. Int denotes the topological interior.

LEMMA 1.4. Let $n \geq 2$ and $K$ be a non-empty, compact, convex subset of $\mathbf{R}^{n}$ such that $0 \notin K$. Let $C=\{\alpha k: \alpha>0, k \in K\}$. Then there is a $k_{0} \in K$ with $k_{0} \notin \operatorname{Int} C$. Then necessarily $k_{0} \notin \operatorname{Int}(\mathbf{R} \cdot K)$. 
Proof of Lemma 1.4. By a separation argument, we see that $k_{0} \notin$ $\operatorname{Int}(\mathbf{R} \cdot K)$ necessarily follows and that $C \neq \mathbf{R}^{n} \backslash\{0\}$. Indeed, choose $f \in\left(\mathbf{R}^{n}\right)^{*}$ and $\lambda$ such that $f(k) \geq \lambda>0$ for all $k \in K$. Then $f(u)>0$ for all $u \in C$ and clearly $C \neq \mathbf{R}^{n} \backslash\{0\}$, since $f$ assumes negative values on $\mathbf{R}^{n} \backslash\{0\}$. Also $\{x: f(x)>\lambda / 2\}$ is an open set not intersecting $\{\alpha k$ : $\alpha \leq 0, k \in K\}$. Hence if $k_{0} \in K \cap \operatorname{Int}(\mathbf{R} \cdot K)$, then $k_{0} \in \operatorname{Int} C$. Of course, pictures of the cone $C$ generated by $K$ make these observations obvious.

Now $C$ is closed in $\mathbf{R}^{n} \backslash\{0\}$. (Let $\left(k_{n}\right)$ be in $K,\left(\alpha_{n}\right)$ in $\mathbf{R}^{+}, x \neq 0$ and suppose $\alpha_{n} k_{n} \rightarrow x$. Then by compactness we may assume $k_{n} \rightarrow k$ for some $k \in K$. Hence $\alpha_{n} \rightarrow \alpha=\|x\| /\|k\|$ and $x=\alpha k \in C$.) We will show that if $K \subset$ Int $C$, then $C$ is open. But this would contradict the fact that $\mathbf{R}^{n} \backslash\{0\}$ is connected, resulting in the conclusion $K \not \subset$ Int $C$. To show that $C$ would be open, let $\alpha k \in C$ where $k \in K$ and $\alpha \in \mathbf{R}^{+}$. If $K \subset \operatorname{Int} C$, there is an open set $U$ with $k \in U \subset C$. Then $\alpha k \in \alpha U \subset C$ and $\alpha U$ is open, completing the proof. (Actually, we only use the case $n=2$; also one could deduce the lemma from this case alone.)

Proof of Lemma 1.3. We have $\|f\|=1, E$ is a finite-dimensional subspace with $\left\|\left.f\right|_{E}\right\|<1$, and $\varepsilon>0$. Let $y_{1} \in X \backslash E$ with $f\left(y_{1}\right)=1$ and $\left\|y_{1}\right\|<1+\varepsilon$. (Such a $y_{1}$ exists because $\|f\|=1$ and $\left\|\left.f\right|_{E}\right\|<1$.) Let $y_{2} \in X \backslash\left(E+\left\langle y_{1}\right\rangle\right)$. Set $Y=E+\left\langle y_{1}, y_{2}\right\rangle$ so that $\operatorname{dim} Y=\operatorname{dim} E+2$ ("dim" denotes "dimension of"). If $\left\|\left.f\right|_{Y}\right\|<1$, we are done; so we now suppose that $\left\|\left.f\right|_{Y}\right\|=1$. Since $B_{Y}$ is compact, $W=\{y \in Y: f(y)=1=$ $\|y\|\}$ is non-empty. Let $V=\{\alpha w+e: \alpha \in \mathbf{R}, w \in W, e \in E\}$. We claim that there exists a $w_{0} \in W$ with $w_{0} \notin$ Int $V$ with respect to the space $Y$. Assuming this claim, choose $\delta>0$ depending on $\varepsilon$ and choose $y \in Y$ with $\left\|y-w_{0}\right\|<\delta$ and $y \notin V$. Thus $\|y\|<1+\delta$ and $f(y)>1-\delta$. Let $\tilde{y}=$ $y / f(y)$; thus $f(\tilde{y})=1$ and $\|\tilde{y}\|<(1+\delta) /(1-\delta)<1+\varepsilon$ if $\delta$ is so chosen. To argue that $\left\|\left.f\right|_{E+\langle y\rangle}\right\|<1$, we suppose this is false. Then ( $E$ $+\langle y\rangle) \cap W \neq \varnothing$, i.e., there exist $e \in E, \alpha \in \mathbf{R}$, and $w \in W$ such that $e+\alpha y=w$. Since $E \cap W=\varnothing, \alpha \neq 0$ and $y=(w-e) / \alpha$ which contradicts the fact that $y \notin V$.

It remains only to prove the claim. Let $\Pi: Y \rightarrow Y / E$ be the quotient map. Note that $Y / E$ is isomorphic to $\mathbf{R}^{2}$. Let $K=\Pi W$, a non-empty, compact, convex set such that $0 \notin K$. By Lemma 1.4, there is a $k_{0} \in K$ with $k_{0} \notin \operatorname{Int}(\mathbf{R} \cdot K)$. Note that $\mathbf{R}_{r} \cdot K=\Pi V$ and since $\Pi$ is an open map, $\Pi($ Int $V) \subset \operatorname{Int}(\Pi V)$. Therefore $k_{0} \notin \Pi(\operatorname{Int} V)$. However $k_{0}=\Pi w_{0}$ for some $w_{0} \in W$, and we conclude that $w_{0} \notin \operatorname{Int} V$. 
2. Closed image characterizations of Tauberian operators. We begin this section by reviewing the definition and discussion of Tauberian operators which are found in [KW]. A remark is also made about the occurrence of a Tauberian operator in the construction of [DFJP] for factoring weakly compact operators. The remainder of the paper will establish new characterizations such as those mentioned in the introduction.

An operator $T$ will always refer to a bounded linear operator from a Banach space $X$ to a Banach space $Y$. We denote the range of $T$ by $R T$ and the null space or kernel of $T$ by $N T$. A bar over a set will always denote its norm closure. For $W \in X, \tilde{W}$ is defined to be the weak*-closure of $W$ in $X^{* *}$. A space is freely identified with its canonical embedding into its double dual. Once again, the term subspace refers to a closed linear subspace.

Definition. An operator $T$ is Tauberian provided $\left(T^{* *}\right)^{-1}(Y)=X$, i.e., $T^{* *} x^{* *} \in Y$ implies $x^{* *} \in X$.

The name Tauberian originates from the fact that a matrix map $A$ : $c \rightarrow c$ has this property as an operator if and only if whenever $x$ is a bounded sequence such that $A x$ converges, then $x$ converges [GW]. Compare this definition with the fact that $T$ is weakly compact if and only if $\left(T^{* *}\right)^{-1}(Y)=X^{* *}([\mathrm{DS}]$, p. 482). Clearly if $X$ is reflexive, $T$ is Tauberian in a trivial way and if $X$ is not reflexive, a Tauberian operator is not weakly compact.

We now summarize the main results from [KW]. Characterization (2) as modified by the final statement, is used in proving our new characterizations.

TheOREM 2.1. Let $T: X \rightarrow Y$ be an operator. The following are equivalent:

(1) $T$ is Tauberian.

(2) NT is reflexive, $\overline{R T^{*}}$ is weak ${ }^{*}$-closed, and $T B_{X}$ is closed.

(3) For all bounded subsets $K$ of $X$, TK relatively weakly compact implies $K$ is relatively weakly compact.

Moreover, the condition $T B_{X}$ is closed may be replaced by the condition $\overline{T B_{X}} \subset R T$.

COROllaRy 2.2. Let $T: X \rightarrow Y$ be an operator.

(A) If $R T$ is closed, then $T$ is Tauberian if and only if $N T$ is reflexive.

(B) If $T$ is one-to-one, then $T$ is Tauberian if and only if $R T^{*}$ is norm-dense in $X^{*}$ and $T B_{X}$ is closed. 
Note that the condition $R T^{*}$ is norm-dense is equivalent to the condition $T^{* *}$ is one-to-one.

An operator property stronger than Tauberian (and previously studied) is now defined.

Definition. An operator $T$ is semi-Fredholm means $R T$ is closed and $N T$ is finite-dimensional.

Kalton and Wilansky also show that if the domain space has no infinite-dimensional reflexive subspace, then $T$ is Tauberian if and only if $T$ is semi-Fredholm. In particular a one-to-one Tauberian operator on such a space (e.g., $l_{1}$ ) is an isomorphism. Therefore a truly interesting Tauberian operator must have a non-reflexive domain $X$ (e.g., $\left.L_{1}\right)$ with an infinite-dimensional reflexive subspace $Z$. In this case, the quotient map $\Pi: X \rightarrow X / Z$ is Tauberian by Corollary 2.2(A).

One source of Tauberian operators (which first aroused the authors' interest in them) is the construction for factoring weakly compact operators in [DFJP]. Given any operator which is not weakly compact and whose range is not closed, this construction produces a non-trivial one-toone Tauberian operator. We now show that the operator so produced has non-reflexive domain. This will elucidate the role Tauberian operators play in this construction.

Given any operator $S: X \rightarrow Y$, the construction in [DFJP] yields a Banach space $Z$ and a one-to-one Tauberian operator $T: Z \rightarrow Y$ such that

$$
S B_{X} \subset T B_{Z} \subset \bigcap_{n=1}^{\infty}\left(2^{n} S B_{X}+2^{-n} B_{Y}\right) .
$$

Using the first containment, one can define an operator from $X$ to $Z$ showing that $S$ factors through $Z$ using $T$. It follows from $(*)$ that $S$ is weakly compact if and only if $T$ is weakly compact. To see this, note that any operator $U: X \rightarrow Y$ is weakly compact if and only if $\widetilde{U B_{X}} \subset Y$. Thus if $S$ is weakly compact, then

$$
\widetilde{T B_{Z}} \subset \bigcap_{n}\left(2^{n} \widetilde{S B_{X}}+2^{-n} B_{Y^{* *}}\right) \subset \bigcap_{n}\left(Y+2^{-n} B_{Y^{* *}}\right)=Y,
$$

and we conclude $T$ is weakly compact. However, since $T$ is Tauberian, $T$ is weakly compact if and only if $Z$ is reflexive. This was mentioned before and can alternatively be seen by Theorem 2.1(3). In other words, the set approximation in $(*)$ preserves weakly compact sets and the Tauberian operator preserves weakly compact sets. This establishes the theorem 
about factoring weakly compact operators through reflexive spaces (modulo the proof of the existence of the Tauberian $T$, of course). Other results can be obtained by using other set properties which are preserved by both the approximation and by Tauberian operators.

The main theorem of this section is now presented; detailing the new characterizations of Tauberian operators in terms of closed images. This leads to a characterization of "hereditary" semi-embeddings and $F_{\sigma}$ embeddings in Corollary 2.4.

THEOREM 2.3. Let $T: X \rightarrow Y$ be a non-zero operator. The following are equivalent:

(1) $T$ is Tauberian.

(2) For all weakly closed bounded subsets $K$ of $X, T K$ is weakly closed.

(3) For all closed bounded convex subsets $K$ of $X, T K$ is closed.

(4) For all closed linear subspaces $E$ of $X, T B_{E}$ is closed.

(5) $N T$ is reflexive and for all closed linear subspaces $E$ of $X, \overline{T B_{E}} \subset T E$.

If $X$ is not reflexive, the following is also equivalent:

(6) $R T$ is infinite-dimensional and for all closed linear subspaces $E$ of $X$, $\overline{T B_{E}} \subset T E$.

REMARK. It follows from the Baire Category Theorem that the condition $\overline{T B_{E}} \subset T E$, in (5) and (6), is equivalent to the condition $T E$ is an $F_{\sigma}$.

We delay the proof in order to put the theorem in context.

This characterization of the operators which preserve closed bounded convex sets complements previous characterizations of the operators which preserve closed sets and those which preserve closed bounded sets. These older results are as follows.

THEOREM. Let $T: X \rightarrow Y$ be a non-zero operator.

(A) $T$ is an isomorphism if and only if for all closed subsets $K$ of $X, T K$ is closed.

(B) $T$ is semi-Fredholm if and only if for all closed bounded subsets $K$ of $X, T K$ is closed.

(C) $T$ is semi-Fredhold or finite rank if and only if for all closed linear subspaces $E$ of $X, T E$ is closed.

In order to prove statement (A), note that if a non-zero $T$ is not one-to-one, by letting $z \in N T \backslash\{0\}$ and letting $x \in X \backslash N T$, one obtains that $T(\{t z+(\arctan t) x: t \in \mathbf{R}\})$ is not closed. Statements (B) and (C) are proved in $[\mathbf{W}]$. The characterization of semi-Fredhold operators in (B) 
differs from Theorem 2.3(2) in changing the norm topology to the weak topology. The theory of Tauberian operators seems to parallel the theory of semi-Fredholm operators in this way.

We now turn to the case of one-to-one operators.

Definition. An operator $T$ is a semi-embedding means $T$ is one-to-one and $T B_{X}$ is closed. $T$ is an $F_{\sigma}$-injection means $T$ is one-to-one and $\overline{T B_{X}} \subset R T$.

As noted above, by the Baire Category Theorem, $\overline{T B_{X}} \subset R T$ if and only if $R T$ is $F_{\sigma}$, hence the name. This agrees with the definition of $F_{\sigma}$-embedding in [BR] in the case that $X$ is separable. By an argument of Saint-Raymond (cf. Prop. 1.6 of [BR]), $T$ is an $F_{\sigma}$-injection if and only if $T$ is a semi-embedding under an equivalent norm on the domain space. In this way, $F_{\sigma}$-injections "remedy" a deficit in semi-embeddings since $F_{\sigma}$ injections are isomorphically invariant and semi-embeddings are not. However both operator properties are not hereditary in the sense that a restriction to a subspace retains the property. For example, $T: l_{\infty} \rightarrow l_{2}$ given by $T x=\left(i^{-1} x(i)\right)_{i=1}^{\infty}$ is a semi-embedding but $\left.T\right|_{c_{0}}$ is not an $F_{\sigma}$-injection. In the one-to-one case, Theorem $2.3(1) \Leftrightarrow(4) \Leftrightarrow(5)$ characterizes operators which have these properties hereditarily.

COROLLARY 2.4. The following are equivalent for an operator $T$ :

(1) $T$ is one-to-one Tauberian.

(2) $T$ restricted to any closed linear subspace is a semi-embedding.

(3) $T$ restricted to any closed linear subspace is in $F_{\sigma}$-injection.

We now proceed to the proof of Theorem 2.3. Assertion (1) $\Rightarrow(2)$ is straight-forward and $(4) \Rightarrow(5)$ amounts to the main result of $\S 1$. The heart of the proof is contained in $(5) \Rightarrow(1)$ which is proved by functional analysis techniques. The proof of $(6) \Rightarrow(5)$ is also non-trivial. Here we use the result of Kadec and Pelczyński that a bounded set which is not relatively weakly compact contains a basic sequence (cf., [S], p. 53). It is phrased for our purpose as follows: In a Banach space a bounded sequence with no weakly convergent subsequence has a basic subsequence. Throughout the proof, $\left[x_{i}\right]$ denotes the closed linear span of $\left\{x_{i}\right\}$ where $i$ runs over the entire index set.

\section{Proof of Theorem 2.3.}

$(1) \Rightarrow(2)$. Assume $T$ is Tauberian, $K$ is a weakly closed bounded subset of $X$, and $y \in$ weak-closure $(T K)$ in order to show $y \in T K$. Since 
$K$ is relatively weak*-compact by Alaoglu's Theorem, $T^{* *}(\tilde{K}) \supset \widetilde{T K}$. Hence, $y=T^{* *} k^{* *}$ for some $k^{* *} \in \tilde{K}$ and by definition of Tauberian, $k^{* *} \in X$. Since $K$ is weakly closed, $k^{* *} \in K$ and $y \in T K$.

(2) $\Rightarrow(3) \Rightarrow(4)$ are trivial.

(4) $\Rightarrow(5)$. We must show (4) implies $N T$ is reflexive. We suppose $N T$ is not reflexive and construct a subspace $E$ of $X$ such that $T B_{E}$ is not closed. Let $x_{0} \in X$ with $\left\|T x_{0}\right\|=1$ and define the subspace $Z=\left[x_{0}\right] \oplus$ $N T$. Let $j:\left[T x_{0}\right] \rightarrow \mathbf{R}$ be the isometry $j\left(\alpha T x_{0}\right)=\alpha$ and define $f \in Z^{*}$ by $f=j \circ T$. Since $Z$ is not reflexive, by Theorem 1.1, there exists a subspace $E$ of $Z$ where $\left.f\right|_{E}$ does not attain its norm, i.e., $f\left(B_{E}\right)$ is not closed. Since $f\left(B_{E}\right)=j\left(T B_{E}\right)$ and $j$ is an isometry, $T B_{E}$ is not closed.

In proving $(5) \Rightarrow(1)$, we use the following standard functional analysis lemma. For $S \subset X^{*}$, we use the notation $S^{\perp}=\left\{F \in X^{* *}: F(f)=0\right.$ for all $f \in S\}$ and ${ }^{\perp} S=\{x \in X: f(x)=0$ for all $f \in S\}$.

Lemma 2.5. Suppose $X$ is a Banach space, $G \in X^{* *}, \mathscr{F}$ is a finite-dimensional subspace of $X^{*}$, and $\varepsilon>0$. Then there is a net $\left(x_{\lambda}\right)$ in $X$ over a directed set $D$ such that $x_{\lambda}$ converges weak* to $G$, and for all $\lambda \in D$, $\left\|x_{\lambda}\right\|<\|G\|(1+\varepsilon)$ and $\left.x_{\lambda}\right|_{\mathscr{F}}=\left.G\right|_{\mathscr{F}}\left(\right.$ i.e., $f\left(x_{\lambda}\right)=G(f)$ for all $\left.f \in \mathscr{F}\right)$.

To prove Lemma 2.5 , let $D$ be the set of finite-dimensional subspaces of $X^{*}$ containing $\mathscr{F}$. Direct $D$ by inclusion. For $\mathscr{G} \in D$, define $x_{\mathscr{G}}$ as follows. Note that $\mathscr{G}^{*}$ is isometric to $X /^{\perp} \mathscr{G}$. (Indeed, since $\mathscr{G}$ is weak*closed, $\mathscr{G}$ can be identified with $\left(X /{ }^{\perp} \mathscr{G}\right)^{*}$ and since $\mathscr{G}$ is reflexive, $\mathscr{G}^{*}$ is identified with $X /{ }^{\perp} \mathscr{G}$.) Consider $\left.G\right|_{\mathscr{G}}$. The identification provides an $x \in X$ (which we label $x_{\mathscr{G}}$ ) such that $\left.G\right|_{\mathscr{G}}=\left.x\right|_{\mathscr{G}}$ and $\|x\|<\left\|\left.G\right|_{\mathscr{G}}\right\|(1+\varepsilon)$. A straightforward check shows that this net satisfies the conclusion.

$(5) \Rightarrow(1)$. By Theorem 2.1, we need only show that $\overline{R T^{*}}$ is weak*closed. We assume $\overline{R T^{*}}$ is not weak*-closed and construct a subspace $E$ of $X$ with $\overline{T B_{E}} \not \subset T E$. Note that weak*-closure $\left(R T^{*}\right)=N T^{\perp}$.

Let $f \in N T^{\perp} \backslash \overline{R T^{*}}$ with $\|f\|=1$. By the Hahn-Banach Theorem, there exists a $G \in X^{* *}$ with $\|G\|<1, G\left(\overline{R T^{*}}\right)=\{0\}$, and $G(f)=\delta>0$. Also, let $h \in R T^{*}$ with $\|h\|=3 / 2$; then there exists an $x_{0} \in B_{X}$ with $h\left(x_{0}\right)=1$. Let $\mathscr{F}=[f, h]$ and let $\varepsilon>0$ so that $\|G\|(1+\varepsilon) \leq 1$ and $\varepsilon<\delta / 5$. By Lemma 2.5, we may choose a directed set $D$ and a net $\left(x_{\lambda}\right)_{\lambda \in D}$ in $B_{X}$ such that $x_{\lambda} \rightarrow G$ weak $^{*}$ and $\left.x_{\lambda}\right|_{\mathscr{F}}=\left.G\right|_{\mathscr{F}}$ for all $\lambda \in D$. Thus $f\left(x_{\lambda}\right)=\delta$ and $h\left(x_{\lambda}\right)=0$ for all $\lambda \in D$.

Let $E=\left[x_{\lambda}+x_{0}\right]_{\lambda \in D}$. We claim that $\frac{1}{2} T x_{0} \in \overline{T B_{E}}$ but $T x_{0} \notin T E$. $T x_{\lambda} \rightarrow T^{* *} G$ weak but $T^{* *} G=G \circ T^{*}=0$. Therefore $T\left(x_{\lambda}+x_{0}\right) \rightarrow T x_{0}$ weakly and $\frac{1}{2} T x_{0} \in \overline{T B_{E}}$. Now suppose $T x_{0}=T e$ for some $e \in E$. Since 
$e-x_{0} \in N T$ and $f, h \in N T^{\perp}, f(e)=f\left(x_{0}\right)$ and $h(e)=h\left(x_{0}\right)=1$. Let $\varepsilon>0$. Because $e \in\left[x_{\lambda}+x_{0}\right]$, there exist $n \in \mathbf{N}$, scalars $c_{1}, \ldots, c_{n}$, and $\lambda_{1}, \ldots, \lambda_{n}$ in $D$ such that $\|z\|<\varepsilon$, where $z=e-\sum_{i=1}^{n} c_{i}\left(x_{\lambda_{l}}+x_{0}\right)$. Observing that $\frac{3}{2} \varepsilon>|h(z)|=\left|1-\sum_{i=1}^{n} c_{i}\right|$ and

$$
\begin{aligned}
\varepsilon & >|f(z)|=\left|f\left(x_{0}\right)-\sum_{i=1}^{n} c_{i}\left(\delta+f\left(x_{0}\right)\right)\right| \\
& =\left|\left(1-\sum_{i=1}^{n} c_{i}\right) f\left(x_{0}\right)-\delta \sum_{i=1}^{n} c_{i}\right| \\
& \geq \delta\left|\sum_{i=1}^{n} c_{i}\right|-\left|1-\sum_{i=1}^{n} c_{i}\right|\left|f\left(x_{0}\right)\right|>\delta\left|\sum_{i=1}^{n} c_{i}\right|-\frac{3}{2} \varepsilon .
\end{aligned}
$$

Therefore $\left|\sum_{i=1}^{n} c_{i}\right| \leq 5 \varepsilon / 2 \delta$ while $\left|1-\sum_{i=1}^{n} c_{i}\right| \leq \frac{3}{2} \varepsilon$ which is impossible since $\varepsilon<\delta / 5$.

$(5) \Rightarrow(6)$. Assume $X$ is not reflexive. Then since $N T$ is reflexive, $R T$ is infinite-dimensional (otherwise $X / N T$ would be finite-dimensional and thus $X$ would be reflexive).

$(6) \Rightarrow(5)$. We must show that if $R T$ is infinite-dimensional and for all subspaces $E$ of $X, \overline{T B_{E}} \subset T E$, then $N T$ is reflexive. We assume $N T$ is not reflexive and $R T$ is infinite-dimensional in order to construct a subspace $E$ with $\overline{T B_{E}} \not \subset T E$.

Since $N T$ is not reflexive, there is a sequence $\left(z_{n}\right)$ in $B_{N T}$ with no weakly convergent subsequence. Consider the quotient map $\Pi: X \rightarrow$ $X / N T$. Since $R T$ and hence $X / N T$ are infinite-dimensional, $X / N T$ contains a basic sequence. In particular, choose $\left(x_{n}\right)_{n=0}^{\infty}$ a sequence in $B_{X}$ with $\left(\Pi x_{n}\right)_{n=0}^{\infty}$ in $X / N T$ and $\left(f_{n}\right)_{n=0}^{\infty}$ in $(X / N T)^{*}$ forming a biorthogonal system. For $n=1,2, \ldots$, define $e_{n}=z_{n}+(1 / n) x_{n}+x_{0}$. Now $\left(e_{n}\right)$ has no weakly convergent subsequence so, by the theorem of Kadec and Pelczyński, there exists a subsequence $\left(e_{n_{i}}\right)$ which is basic.

Let $E=\left[e_{n_{i}}\right]$. We claim that $\left(\frac{1}{3}\right) T x_{0} \in \overline{T B_{E}}$ but $T x_{0} \notin T E$. Since $T e_{n_{i}}=\left(1 / n_{i}\right) T x_{n_{i}}+T x_{0} \rightarrow T x_{0}$ and $\left\|e_{n_{i}}\right\| \leq 3$ for all $i$, it is clear that $\left(\frac{1}{3}\right) T x_{0} \in \overline{T B_{E}}$. Now suppose $T x_{0}=T e$ for some $e \in E$; choose $c_{1}, c_{2}, \ldots$ so that $e=\sum_{i=1}^{\infty} c_{l} e_{n_{i}}$. Since $e-x_{0} \in N T=N \Pi$,

$$
\left.\Pi\left(e-x_{0}\right)=\sum_{i=1}^{\infty} c_{i}\left(\frac{1}{n_{i}}\right) \Pi x_{n_{i}}+\Pi x_{0}\right)-\Pi x_{0}=0 .
$$

Applying $f_{0}$ to this equation yields $\left(\sum_{i=1}^{\infty} c_{i}\right)-1=0$. For $i=1,2, \ldots$, applying $f_{n_{i}}$ yields $c_{i} / n_{i}=0$. This contradiction completes the entire proof. 


\section{REFERENCES}

[B] J. Bourgain, On dentability and the Bishop-Phelps property, Israel J. Math., 28 (1977), 265-271.

[BR] J. Bourgain and H. P. Rosenthal, Applications of the theory of semi-embeddings to Banach space theory, J. Functional Analysis, 52 (1983), 149-188.

[DFJP] W. J. Davis, T. Figiel, W. B. Johnson and A. Pelczyński, Factoring weakly compact operators, J. Functional Analysis, 17 (1974), 311-327.

[DS] N. Dunford and J. Schwartz, Linear Operators Part I, Interscience, New York, 1958.

[GW] D. J. H. Garling and A. Wilansky, On a summability theorem of Berg, Crawford and Whitley, Proc. Cambridge Philos. Soc., 71 (1972), 495-497.

[J1] R. C. James, Reflexivity and the sup of linear functionals, Israel J. Math., 13 (1972), 289-300.

[J2] _ A counterexample for a sup theorem in normed spaces, Israel J. Math., 9 (1971), 511-512.

[KW] N. Kalton and A. Wilansky, Tauberian operators on Banach spaces, Proc. Amer. Math. Soc., 57 (1976), 251-255.

[NR] R. Neidinger and H. P. Rosenthal, Characterizing Tauberian operators by closed images, Longhorn Notes, 1982-1983.

[S] I. Singer, Bases in Banach Spaces II, Springer-Verlag, New York, 1981.

[W] A. Wilansky, Semi-Fredholm maps of FK spaces, Math. Z., 144 (1975), 9-12.

Received November 1, 1983. This is part of the first author's $\mathrm{Ph}$. D. dissertation prepared at The University of Texas at Austin under the supervision of the second author. The research was supported in part by NSF-MCS- 8303534 .

THE UNIVERSITY OF TEXAS

Austin, TX 78712 


\title{
PACIFIC JOURNAL OF MATHEMATICS EDITORS
}

DONALD BABBITT (Managing Editor)
University of California
Los Angeles, CA 90024
CHARLEs R. DEPrIMA
California Institute of Technology
Pasadena, CA 91125
R. FINN
Stanford University
Stanford, CA 94305

DoNAld BABBitT (Managing Editor)

Hermann FlaschKa

University of Arizona

Tucson, AZ 85721

RAmesh A. Gangolli

University of Washington

Seattle, WA 98195

ROBION KIRBY

University of California

Berkeley, CA 94720

C. C. MOORE

University of California

Berkeley, CA 94720

\author{
Hugo Rossi \\ University of Utah \\ Salt Lake City, UT 84112 \\ H. SAMELSON \\ Stanford University \\ Stanford, CA 94305 \\ HAROLD STARK \\ University of California, San Diego \\ La Jolla, CA 92093
}

ASSOCIATE EDITORS

R. ARENS

E. F. BECKENBACH
(1906-1982)

B. H. NEUMANN

F. WOLF

K. Yoshida

\section{SUPPORTING INSTITUTIONS}

UNIVERSITY OF ARIZONA

UNIVERSITY OF BRITISH COLUMBIA

CALIFORNIA INSTITUTE OF TECHNOLOGY

UNIVERSITY OF CALIFORNIA

MONTANA STATE UNIVERSITY

UNIVERSITY OF NEVADA, RENO

NEW MEXICO STATE UNIVERSITY

OREGON STATE UNIVERSITY
UNIVERSITY OF OREGON

UNIVERSITY OF SOUTHERN CALIFORNIA

STANFORD UNIVERSITY

UNIVERSITY OF HAWAII

UNIVERSITY OF TOKYO

UNIVERSITY OF UTAH

WASHINGTON STATE UNIVERSITY

UNIVERSITY OF WASHINGTON 


\section{Pacific Journal of Mathematics}

\section{Vol. 118, No. $1 \quad$ March, 1985}

Dan Amir, On Jung's constant and related constants in normed linear spaces ...1 Abdul Aziz, On the location of the zeros of certain composite polynomials . . 17 Joseph Barback, On hereditarily odd-even isols and a comparability of

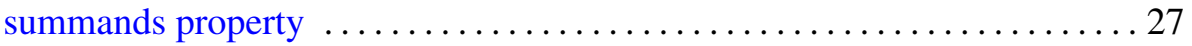

Matthew G. Brin, Klaus Johannson and Peter Scott, Totally peripheral 3-manifolds ........................................ 37

Robert F. Brown, A topological bound on the number of distinct zeros of an

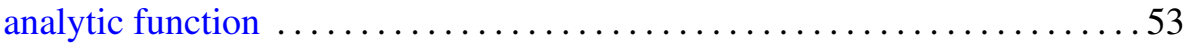

K. C. Chattopadhyay, Not every Lodato proximity is covered .......... 59

Beverly Diamond, Some properties of almost rimcompact spaces . .......63 63

Manfred Dugas and Rüdiger Göbel, On radicals and products . ......... 79

Abdelouahab El Kohen, A hyperbolic problem .................. 105

Harry Gonshor, Remarks on the Dedekind completion of a nonstandard model of the reals ................................... 117

William H. Kazez, On equivalences of branched coverings and their action

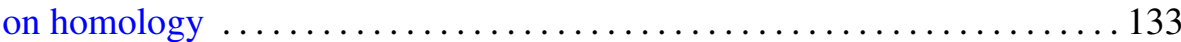

Darrell Conley Kent, On the Wallman order compactification .......... 159

Martin Andrew Magid, Lorentzian isoparametric hypersurfaces . . . . . . 165

Milan Miklavčič, Stability for semilinear parabolic equations with noninvertible linear operator

Richard Dean Neidinger and Haskell Paul Rosenthal, Norm-attainment of linear functionals on subspaces and characterizations of Tauberian operators

Johannes Vermeer, Closed subspaces of $H$-closed spaces 